\title{
Inhibition of PIKfyve using YM201636 suppresses the growth of liver cancer via the induction of autophagy
}

\author{
JIU-ZHOU HOU ${ }^{1}$, ZHUO-QING XI ${ }^{1}$, JIE NIU ${ }^{1}$, WEI LI ${ }^{1}$, XIAO WANG $^{1}$, CHAO LIANG $^{1}$, \\ HUA SUN $^{1}$, DONG FANG ${ }^{1}$ and SONG-QIANG XIE ${ }^{2}$ \\ ${ }^{1}$ Institute for Innovative Drug Design and Evaluation; ${ }^{2}$ Institute of Chemical Biology, \\ School of Pharmacy, Henan University, Kaifeng, Henan 475004, P.R. China
}

Received May 9, 2018; Accepted December 6, 2018

DOI: $10.3892 / o r .2018 .6928$

\begin{abstract}
Liver cancer is among the most common types of cancer worldwide. The aim of the present study was to investigate whether the phosphatidylinositol-3-phosphate 5-kinase (PIKfyve) inhibitor, YM201636, exerts anti-proliferative effects on liver cancer. The methods used in the present study included MTT assay, flow cytometry, western blot analysis and an allograft mouse model of liver cancer. The results revealed that YM201636 inhibited the proliferation of HepG2 and Huh-7 cells in a dose-dependent manner. HepG2 and Huh-7 cells exhibited strong monodansylcadaverine staining following treatment with YM201636. Accordingly, YM201636 treatment increased the expression of the autophagosome-associated marker protein microtubule-associated 1A/1B light chain 3-II in HepG2 and Huh-7 cells. The autophagy inhibitor 3-methyladenine attenuated the inhibitory effects of YM201636 on liver cancer cell proliferation. Further in vivo analysis revealed that YM201636 (2 mg/ $\mathrm{kg})$ inhibited tumor growth without notable systemic toxicity. Mechanistic experiments demonstrated that YM201636 induced-autophagy is dependent upon epidermal growth factor receptor (EGFR) overexpression in HepG2 and Huh-7 cells. Collectively, these results suggested that the PIKfyve inhibitor YM201636 may inhibit tumor growth by promoting EGFR expression. This indicates that PIKfyve may be a potential therapeutic target for the treatment of liver cancer.
\end{abstract}

Correspondence to: Dr Dong Fang, Institute for Innovative Drug Design and Evaluation, School of Pharmacy, Henan University, Pharmacy Building, N. Jinming Avenue, Kaifeng, Henan 475004, P.R. China

E-mail: emailfangdong@163.com

Dr Song-Qiang Xie, Institute of Chemical Biology, School of Pharmacy, Henan University, Pharmacy Building, N. Jinming Avenue, Kaifeng, Henan 475004, P.R. China

E-mail: xiesq@vip.henu.edu.cn

Key words: YM201636, autophagy, epidermal growth factor receptor, hepatoma

\section{Introduction}

Liver cancer is one of the most common types of cancer worldwide, ranking as the third leading cause of cancer-associated mortality (1). Despite the great advances in the use of modern surgical techniques in combination with chemotherapy, the overall 5-year survival rate for patients with liver cancer remains poor (2). Therefore, novel strategies for the anticancer therapy of liver cancer are urgently required.

Phosphatidylinositol-3-phosphate 5-kinase (PIKfyve) is a lipid kinase that phosphorylates phosphatidylinositol-3-phosphate (PI3P) to generate phosphatidylinositol 3,5-bisphosphate [PtdIns(3,5)P2] or phosphatidylinositol 5-phosphate (PtdIns5P) $(3,4)$. PtdIns (3,5)P2 and PtdIns5P have been proposed to be involved in several cellular functions, including vesicular trafficking, ion channel activation and epidermal growth factor receptor (EGFR) signaling (5-7). In addition, accumulating evidence has suggested that PIKfyve is involved in oncogenesis and cancer cell migration $(8,9)$, and knockdown of PIKfyve resulted in a significant decrease in cancer cell migration (10). Therefore, the potential role of PIKfyve inhibition in anticancer therapy was explored in the present study. Recent studies demonstrated that inhibition of PIKfyve activity using the inhibitor YM201636 led to a strong reduction in cell proliferation in multiple cancers $(8,11)$; however, whether PIKfyve inhibition could be applied for anticancer therapy of liver cancer remains unknown. Therefore, the aim of the present study was to investigate the antitumor activity of the PIKfyve inhibitor, YM201636, in liver cancer.

\section{Materials and methods}

Reagents and antibodies. RPMI-1640 and fetal bovine serum (FBS) were purchased from Gibco (Thermo Fisher Scientific, Inc., Waltham, MA, USA). MTT and monodansylcadaverine (MDC) were purchased from Sigma-Aldrich (Merck KGaA, Darmstadt, Germany). YM201636 (cat. no. sc-204193) was obtained from Santa Cruz Biotechnology, Inc. (Dallas, TX, USA). Monoclonal mouse anti-human EGFR antibody (cat. no. sc-71034; 1:200), monoclonal mouse anti-human phospho (p)-EGFR antibody (cat. no. sc-81490; 1:200), monoclonal mouse anti-human $\beta$-actin antibody (cat. no. sc-47778; 1:1,000) were all purchased from 
Santa Cruz Biotechnology, Inc. Polyclonal rabbit anti-human microtubule associated protein 1 light chain 3 (LC3) A/B antibody (cat. no. 14600-1-AP; 1:1,000) was purchased from ProteinTech Group, Inc. (Chicago, IL, USA). The horseradish peroxidase (HRP)-conjugated secondary antibodies including goat anti-rabbit IgG (cat. no. sc-2054; 1:1,000) and goat anti-mouse IgG (cat. no. sc-2973; 1:1,000) were also purchased from Santa Cruz Biotechnology, Inc.

Cell lines and cell culture. All cell lines (HepG2, Huh-7 and H22) used in the present study were purchased from the Cell Bank of Type Culture Collection of Chinese Academy of Sciences (Shanghai, China). These cells were cultured in RPMI-1640 medium supplemented with 10\% FBS, $100 \mathrm{U} / \mathrm{ml}$ penicillin and $100 \mu \mathrm{g} / \mathrm{ml}$ streptomycin, at $37^{\circ} \mathrm{C}$ in a humidified incubator containing $5 \% \mathrm{CO}_{2}$.

Transient transfections. HepG2 and Huh-7 cells were transiently transfected with pcDNA3.1-epidermal growth factor receptor (EGFR) plasmid or control pcDNA3.1 plasmid (Shanghai GeneChem Co., Ltd., Shanghai, China) using Lipofectamine ${ }^{\circledR} 2000$ reagent (Invitrogen; Thermo Fisher Scientific, Inc.) according to the manufacturer's instructions. Briefly, the cells were seeded in 6-well plates at $5 \times 10^{5} /$ well and cultured at $37^{\circ} \mathrm{C}$ in a humidified atmosphere with $5 \% \mathrm{CO}_{2}$. The subsequent day, when the cells were $\sim 70 \%$ confluent, they were transfected with a mixture of $3 \mu$ l Lipofectamine ${ }^{\circledR} 2000$ and $2 \mu \mathrm{g}$ plasmids. At $4 \mathrm{~h}$ post-transfection, the cell culture medium was replaced with RPMI-1640 medium. After $24 \mathrm{~h}$, these transfected cells were collected to perform MDC staining or western blot analysis.

MTT assay. Cells were seeded in 96-well plates at an initial density of $4 \times 10^{3}$ cells/well in $90 \mu \mathrm{l}$ medium and cultured overnight. As cells reached $30 \%$ confluence, various concentrations of YM201636 $(0.1,0.2,0.5,1,2$ and $5 \mu \mathrm{M})$ were added to the cells, which were then incubated for $24 \mathrm{~h}$. Subsequently, $50 \mu \mathrm{l}$ $1 \mathrm{mg} / \mathrm{ml}$ of MTT (Sigma-Aldrich; Merck KGaA) was added to each well for $4 \mathrm{~h}$. Then, the supernatant was removed and $100 \mu \mathrm{l}$ DMSO was subsequently added to solubilize the crystal products at room temperature for $10 \mathrm{~min}$. The optical density (OD) was measured at a wavelength of $490 \mathrm{~nm}$ using a microplate reader (BioTek Instruments, Inc., Winooski, VT, USA). The growth inhibition ratio was calculated as follows: Growth inhibition ratio $(\%)=\left(\mathrm{OD}_{\text {control }}-\mathrm{OD}_{\text {drug }}\right) / \mathrm{OD}_{\text {control }} \times 100$; where $\mathrm{OD}_{\text {control }}$ is the $\mathrm{OD}$ of the group treated with vehicle. The experiments were repeated at least 3 times. To verify whether the mechanism of autophagy promoted cell survival or cell death, HepG2 and Huh-7 cells were pretreated with 5 mM 3-methyladenine (3-MA) for 30 min followed by cotreatment with $2 \mu \mathrm{M}$ YM201636 for $24 \mathrm{~h}$. Then, an MTT assay was performed. The growth inhibition ratio was calculated as follows: Growth inhibition ratio $(\%)=\left(\mathrm{OD}_{\text {control }}-\mathrm{OD}_{\text {drug }}\right) / \mathrm{OD}_{\text {control }} \times 100$; where $\mathrm{OD}_{\text {control }}$ is the $\mathrm{OD}$ of the group treated with vehicle. To examine the effects of EGFR overexpression on cell growth, HepG2 and Huh-7 cells (80\% confluence) seeded in a 6-well plate were transfected with pcDNA3.1-EGFR or control pcDNA3.1 plasmids using Lipofectamine ${ }^{\circledR} 2000$. After $24 \mathrm{~h}$, the cells were harvested and seeded into 96-well plates at a density of $4 \times 10^{3}$ cells/well for another $24 \mathrm{~h}$. An MTT assay was performed to determine the OD value of each group. During incubation, EGF was not added to the culture medium to avoid EGFR activation.

Flow cytometry. MDC, a specific marker for autophagic vacuoles, was used to examine whether YM201636 induced autophagy. HepG2 and Huh-7 cells were grown on coverslips in a 6-well plate overnight at $3 \times 10^{5} /$ well, and then treated with 2 or $5 \mu \mathrm{M}$ of YM201636 for $24 \mathrm{~h}$. The cells were collected and washed with ice-cold PBS 3 times, then incubated with $50 \mu \mathrm{M}$ $\mathrm{MDC}$ at $37^{\circ} \mathrm{C}$ for $30 \mathrm{~min}$. The stained cells were washed, fixed with $4 \%$ paraformaldehyde for $10 \mathrm{~min}$ at room temperature and analyzed with the imaging flow cytometer FlowSight ${ }^{\circledR}$ (Amnis ${ }^{\circledR}$; Merck KGaA). A laser set at $405 \mathrm{~nm}$ was used for excitation. Bright field and MDC images (green fluorescence) were collected in channels 1 and 8 , respectively. The stained cells $\left(1 \times 10^{4} /\right.$ sample) were analyzed using IDEAS version 6.0 software (Merck KGaA).

Western blot analysis. The total proteins were isolated from cancer cells or allograft tumors using radio immunoprecipitation assay buffer (Beyotime Institute of Biotechnology, Haimen, China) following the manufacturer's instructions. The protein concentration was determined using a bicinchoninic acid assay kit (Pierce; Thermo Fisher Scientific, Inc.). Samples were denatured in $5 \mathrm{X} \mathrm{SDS}$-sample buffer at $95^{\circ} \mathrm{C}$ for $5 \mathrm{~min}$. Total proteins ( $40 \mu \mathrm{g} /$ well) were separated using SDS-PAGE on $10 \%$ gels for EGFR detection or $12 \%$ gels for LC3 detection. Following separation, the protein was transferred onto polyvinylidene difluoride membranes. Subsequently, the membranes were blocked using 5\% non-fat milk in TBS-Tween (TBS-T) at room temperature for $1 \mathrm{~h}$. Following blocking, membranes were incubated with corresponding primary antibodies overnight at $4^{\circ} \mathrm{C}$, then washed 3 times with TBST and incubated with the appropriate HRP-conjugated secondary antibody, and then washed 3 times with TBST. Proteins were detected using the enhanced chemiluminescence plus reagents (Beyotime Institute of Biotechnology). The western blots were visualized using a FluroChem E Imager (Protein Simple, San Jose, CA, USA). Quantity One Software (Quantity One 462; Bio-Rad Laboratories, Inc., Hercules, CA, USA) was used to calculate the alteration of corresponding protein expression.

Evaluation of antitumor effects in vivo. Male BALB/c mice (weighing 20-25 g; aged 5 weeks) were purchased from the Laboratory Animal Center of Henan (Zhengzhou, China). All animal procedures were performed with the approval of the Institutional Animal Care and Use Committee of Henan University (Kaifeng, China). For the development of solid tumors, mice were subcutaneously injected with $2 \times 10^{6} \mathrm{H} 22$ cells. At 1 day after inoculation, the mice were randomly divided into the control and YM201636 groups, which were treated with 5\% DMSO or $2 \mathrm{mg} / \mathrm{kg}$ YM201636, respectively, via oral administration once daily for 7 consecutive days. The mice were anesthetized using ether for $\sim 30 \mathrm{sec}$ via the respiratory route, and the heart rate and respiratory rate were monitored to ensure the animals were simply anesthetized. Then the mice were euthanized by cervical dislocation, and solid tumors were isolated and weighed. Meanwhile, the 

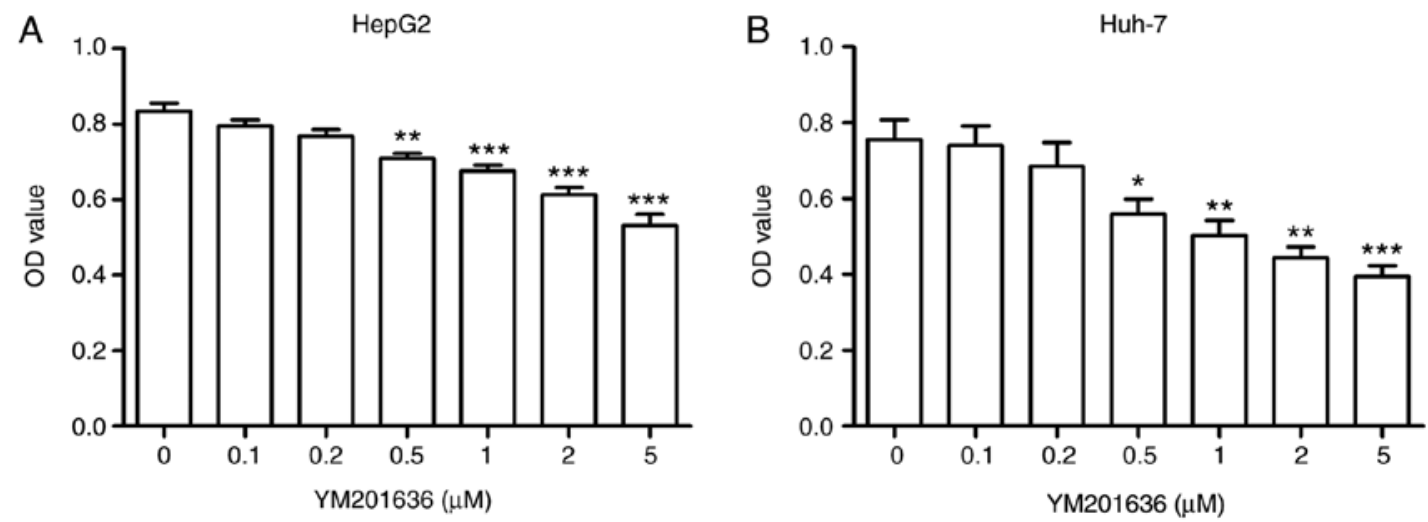

Figure 1. YM201636 inhibits liver cancer cell proliferation in a dose-dependent manner. MMT assay was performed using (A) HepG2 and (B) Huh-7 cells. ${ }^{*} \mathrm{P}<0.05,{ }^{* *} \mathrm{P}<0.01,{ }^{* * * *} \mathrm{P}<0.001$, one-way analysis of variance followed by a Dunnett's test, $\mathrm{n}=5 / \mathrm{group}$.

heart, liver, kidney, lung, and spleen of the mice were collected and weighed on the last day. The organ weight index was investigated for systemic toxicity evaluation as follows: Organ index $(\%)=($ organ weight/bodyweight $) \times 100$.

Statistical analysis. Statistical analyses were performed using GraphPad Prism 5 software for Windows (GraphPad Software, Inc., La Jolla, CA, USA). All data are expressed as the mean \pm standard error. A two-tailed unpaired t-test was used for the comparison of the mean values between two groups. One-way analysis of variance (ANOVA) followed by a Dunnett's test or two-way ANOVA followed by a Bonferroni post hoc test was used for multiple comparisons. $\mathrm{P}<0.05$ was considered to indicate a statistically significant difference.

\section{Results}

Effects of YM201636 on HepG2 and Huh-7 cell viability. In order to determine whether the PIKfyve inhibitor YM201636 affects the cell viability of hepatoma, HepG2 and Huh-7 cells were cultured and incubated in the presence of increasing concentrations of the drug for $24 \mathrm{~h}$. Cell viability rates were then detected using an MTT assay. Following incubation of HepG2 and Huh-7 cells with $0.1,0.2,0.5,1,2$ and $5 \mu \mathrm{M}$ YM201636 for $24 \mathrm{~h}$, YM201636 reduced the HepG2 and Huh-7 cell viability in a dose-dependent manner (Fig. 1). The data indicated that YM201636 may inhibit the cell growth of liver cancer cell lines.

YM201636 induces autophagy in liver cancer cell lines. Previous studies have demonstrated that knockdown or inhibition of PIKfyve induces secretory autophagy in multiple cell lines $(12,13)$. Therefore, the present study investigated whether decreased cell viability was associated with autophagy in liver cancer cell lines using an MDC staining assay. Following treatment with 2 or $5 \mu \mathrm{M}$ YM201636 for $24 \mathrm{~h}, \mathrm{HepG} 2$ and Huh-7 cells exhibited strong MDC staining (Fig. 2), with enhanced fluorescence in cells treated with $5 \mu \mathrm{M}$ YM201636. Additionally, the percentage of autophagic cells in the population was increased in HepG2 and Huh-7 cells treated with YM201636 for $24 \mathrm{~h}$ (Fig. 2). To further confirm whether YM201636 induces autophagy in liver cancer cell lines, the ratio of LC3I to LC3II was determined, an established autophagosome-related marker $(14,15)$. The results demonstrated that YM201636 significantly promoted the conversion of LC3I to LC3II in HepG2 and Huh-7 cells (Fig. 3). To verify whether the mechanism of autophagy promoted cell survival or cell death, the autophagy inhibitor 3-MA was applied to inhibit YM201636 induced-autophagy in HepG2 and Huh-7 cells; 3-MA inhibits autophagy by blocking autophagosome formation via the inhibition of type III phosphatidylinositol 3-kinases (PI3Ks) (16,17). Co-treatment with 5 mM 3-MA for $24 \mathrm{~h}$ attenuated the inhibitory effects of YM201636 on liver cancer cell lines (Fig. 4). Collectively, these results suggested that YM201636 inhibits the growth of HepG2 and Huh-7 cells via the induction of autophagy.

YM201636 induced-autophagy depends on EGFR overexpression. It has been previously reported that PIKfyve inhibition blocks the lysosomal degradation of EGFR, resulting in increased expression levels of EGFR in MCF-10A cells (18), and overexpression of inactive EGFR has been demonstrated to be associated with autophagy (19). Therefore, the present study investigated whether YM201636-induced autophagy is dependent upon EGFR overexpression. To test this hypothesis, the total protein expression of EGFR was determined in HepG2 and Huh-7 cells treated with YM201636. Western blotting demonstrated that the total protein expression levels of EGFR were significantly upregulated in HepG2 and Huh-7 cells treated with $2 \mu \mathrm{M}$ YM201636 for $24 \mathrm{~h}$ (Fig. 5). To investigate whether the increased EGFR was activated in HepG2 and Huh-7 cells treated with YM201636, the phosphorylation levels of EGFR at Tyr ${ }^{1068}$, an indicator of EGFR activation, were examined. As shown in Fig. 5, the phosphorylation levels of EGFR at Tyr ${ }^{1068}$ were notably unaffected in HepG2 and Huh-7 cells treated with YM201636. The results suggested that the increased total protein constituted inactive EGFR.

To further investigate the hypothesis that inactive EGFR mediates autophagy in HepG2 and Huh-7 cells, HepG2 and Huh-7 cells were transfected with the pcDNA3.1-EGFR plasmid with no EGF treatment. At $24 \mathrm{~h}$ after transfection, the expression of EGFR was increased significantly (Fig. 6). Subsequently, whether overexpression of EGFR was able to affect autophagy in liver cancer cells was examined. As demonstrated in Fig. 7, HepG2 and Huh-7 cells transfected with EGFR exhibited strong MDC staining and an increase in 
A

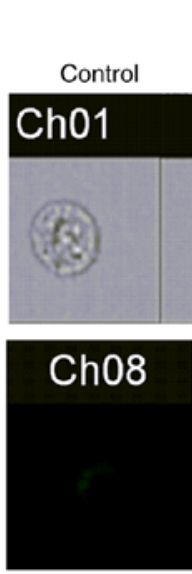

C

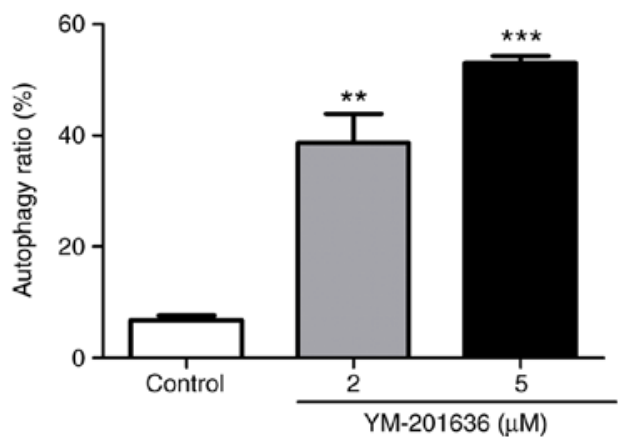

B
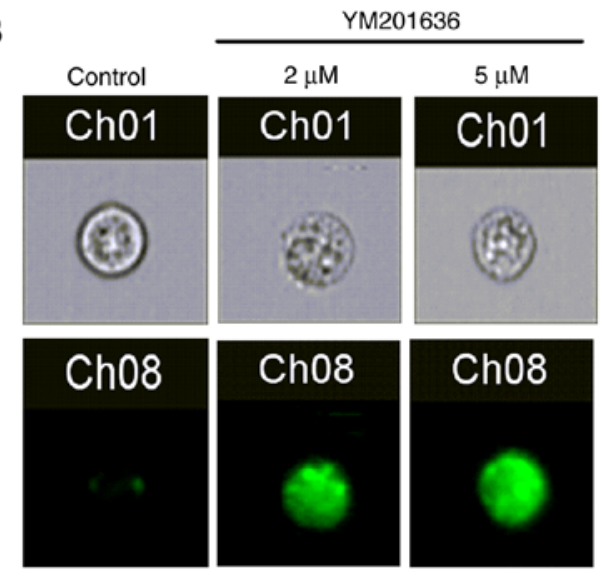

Huh-7

D

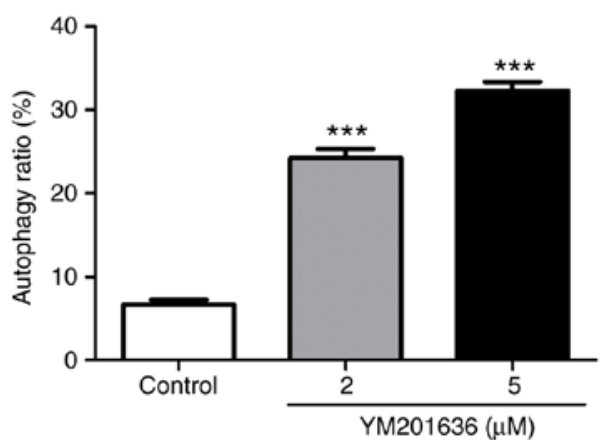

Figure 2. YM201636 induces autophagy in HepG2 and Huh-7 cells. Representative monodansylcadaverine staining of (A) HepG2 and (B) Huh-7 cells. Analysis of the autophagy ratio of (C) HepG2 and (D) Huh-7 cells treated with YM201636. ${ }^{* *} \mathrm{P}<0.01,{ }^{* * *} \mathrm{P}<0.001$, one-way analysis of variance followed by a Dunnett's test, $\mathrm{n}=5 /$ group.

A
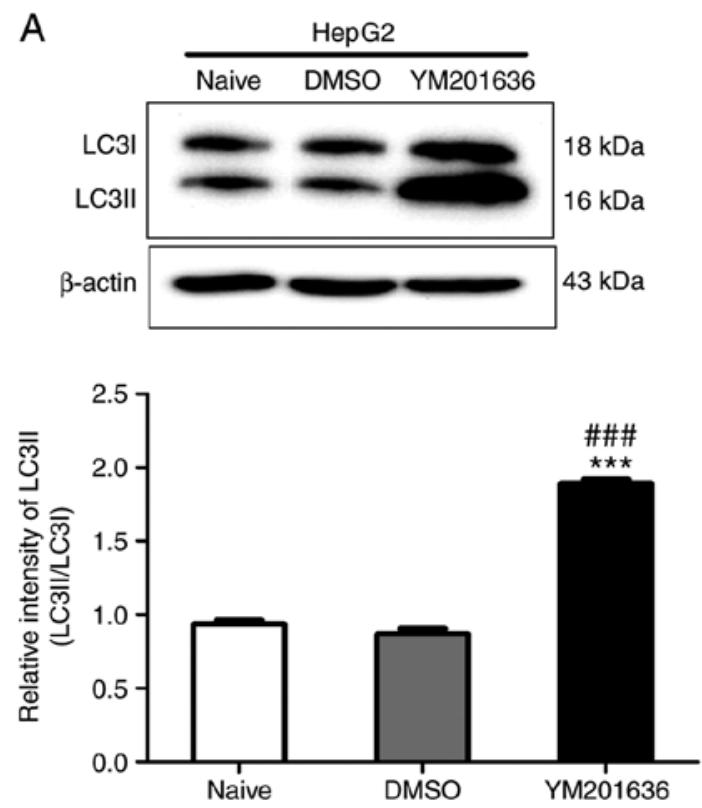

B
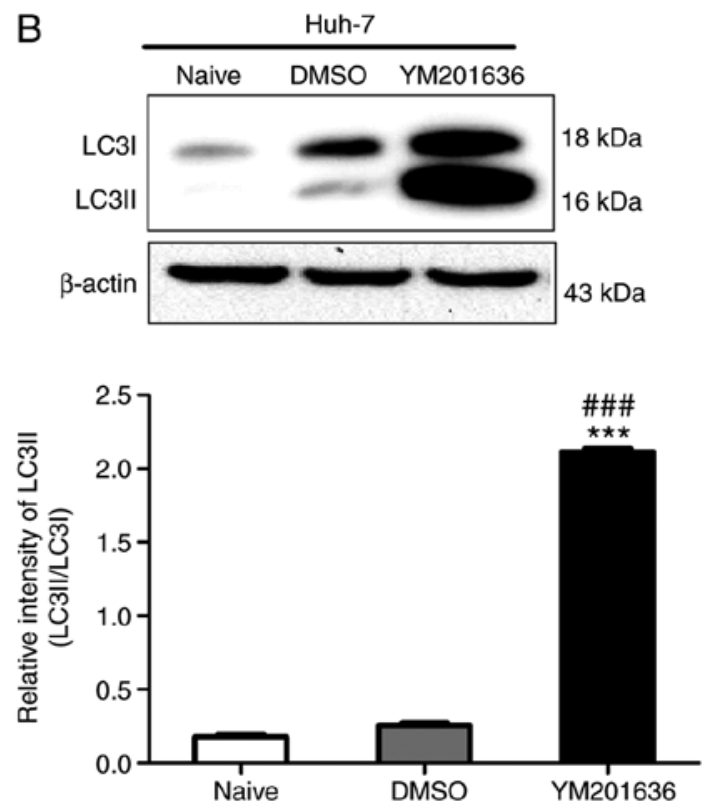

Figure 3. YM201636 significantly increased the levels of LC3II. (A) HepG2 and (B) Huh-7 cells. $\beta$-actin was used as an internal control. Naïve cells were untreated. ${ }^{* * *} \mathrm{P}<0.001$ vs. DMSO group, ${ }^{\# \# \#} \mathrm{P}<0.001$ vs. naive group, one-way analysis of variance followed by a Dunnett's test, $\mathrm{n}=5 /$ group. $\mathrm{DMSO}$, dimethyl sulfoxide; LC3, microtubule associated protein 1 light chain 3.

the proportion of autophagic cells. Additionally, overexpression of EGFR also promoted the transformation of LC3I into LC3II in HepG2 and Huh-7 cells (Fig. 8). Furthermore, the effects of EGFR overexpression on liver cancer cell growth were determined. Overexpression of EGFR in HepG2 and Huh-7 cells inhibited cell proliferation (Fig. 9). The results indicated that YM201636 induced-autophagy was dependent on EGFR overexpression in HepG2 and Huh-7 cells. 

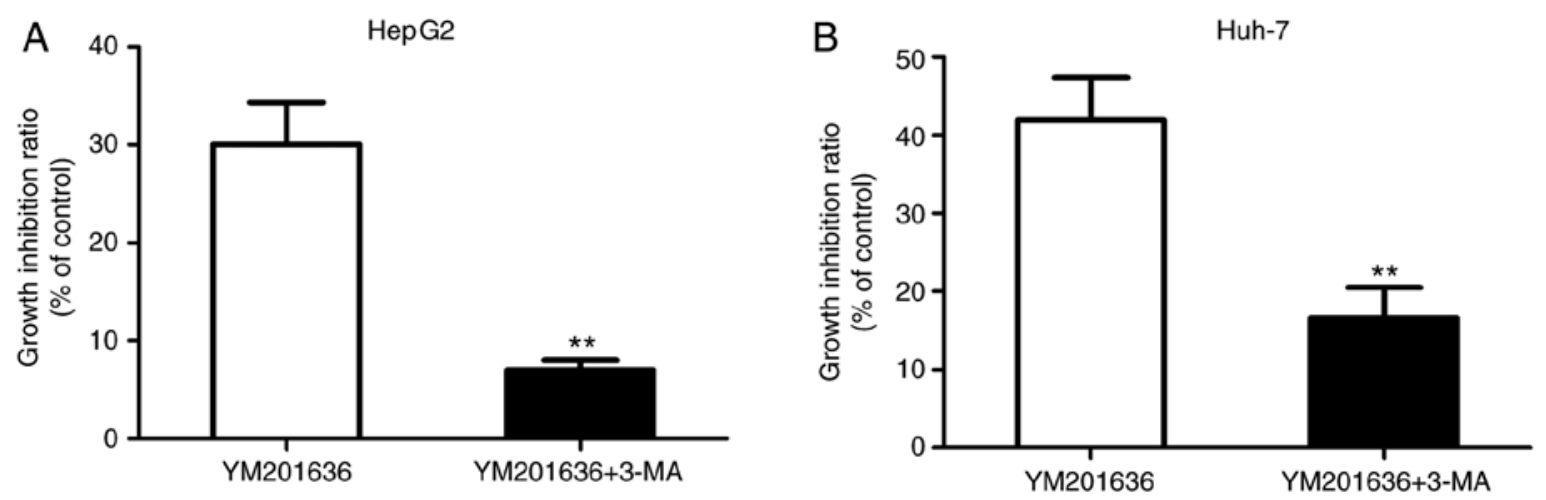

Figure 4. 3-MA reverses YM201636-induced inhibition of liver cancer cell proliferation. MTT assay in (A) HepG2 and (B) Huh-7 cells treated with 5 mM 3-MA and YM201636. ${ }^{* *} \mathrm{P}<0.01$ vs. YM201636 group, two-tailed unpaired t-test, n=5/group. 3-MA, 3-methyladenine.
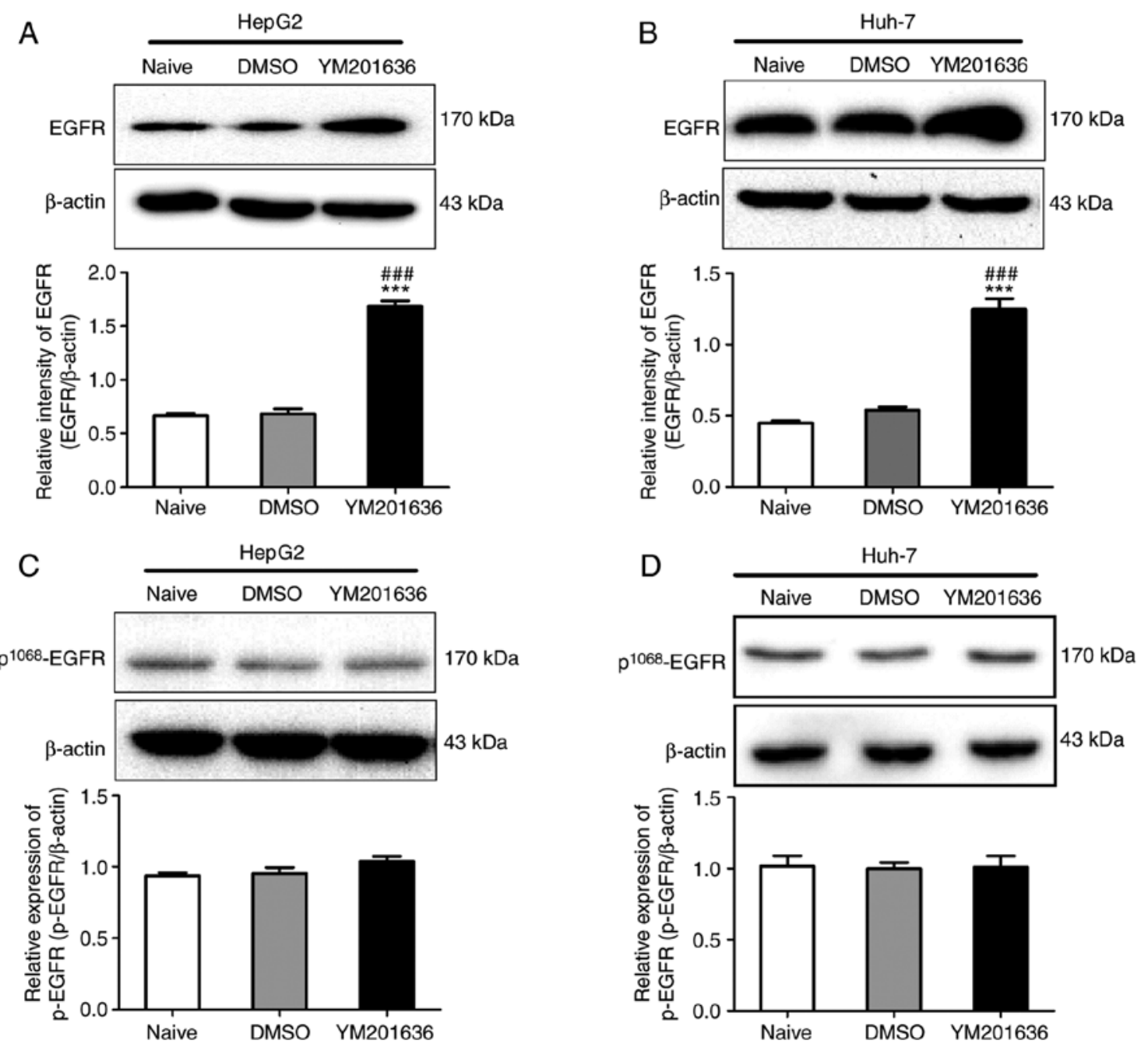

Figure 5. YM201636 significantly increases the total protein expression of EGFR. Western blot analysis was performed to detect EGFR in (A) HepG2 and (B) Huh-7 cells and p-EGFR (Tyr ${ }^{1068}$ ) in (C) HepG2 and (D) Huh-7 cells following treatment with YM201636. $\beta$-actin was used as an internal control. Naïve cells were untreated. ${ }^{* * *} \mathrm{P}<0.001$ vs. DMSO group, ${ }^{\# \# \#} \mathrm{P}<0.001$ vs. naive group, one-way analysis of variance followed by a Dunnett's test, $\mathrm{n}=5 /$ group. DMSO, dimethyl sulfoxide; EGFR, epidermal growth factor receptor; p-, phospho-.

YM201636 inhibits the in situ growth of hepatoma in vivo. To further evaluate the antitumor activity of the PIKfyve inhibitor, YM201636, in vivo, an allograft model of mouse liver cancer was established by subcutaneously injecting H22 cells (mouse hepatoma cell line) into BALB/c mice. Transplantation of $\mathrm{H} 22$ cells into BALB/c mice induced the in situ formation of tumors. Following treatment with $2 \mathrm{mg} / \mathrm{kg}$ YM201636 for 7 consecutive days, the tumor weight of the YM201636 group was lower than that of the negative control group. For example, the mean weight of tumors in the YM201636-treated group 
A
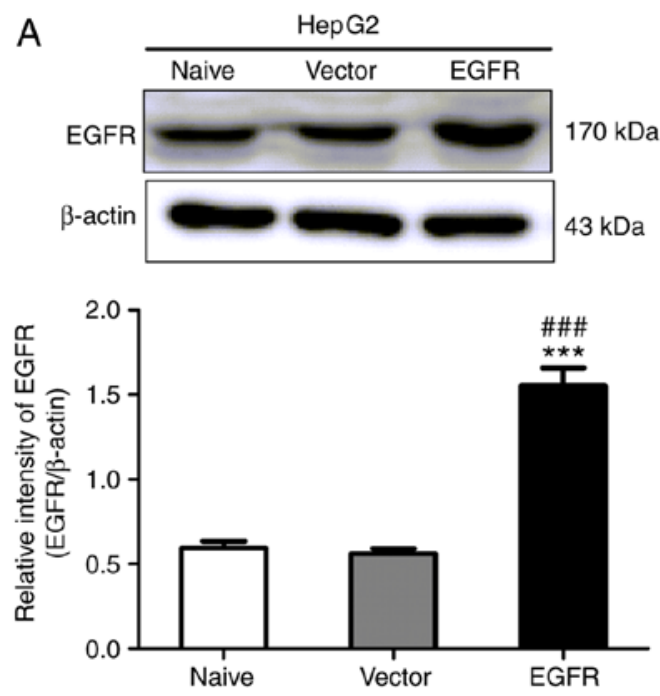

B
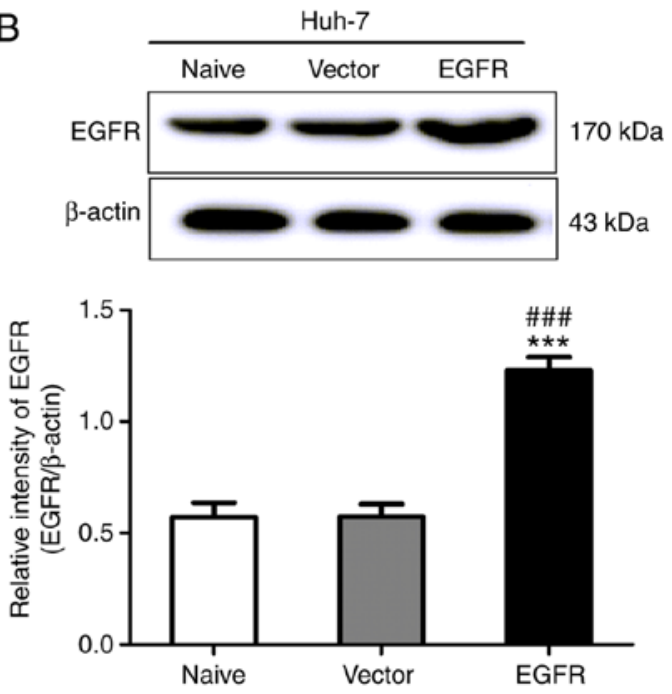

Figure 6. EGFR overexpression using pcDNA3.1-EGFR plasmid. EGFR protein is increased in (A) HepG2 and (B) Huh-7 cells transfected with. $\beta$-actin was used as an internal control. Naïve cells were untreated. ${ }^{* * *} \mathrm{P}<0.001$ vs. vector group, ${ }^{\# \# \#} \mathrm{P}<0.001$ vs. naive group, one-way analysis of variance followed by a Dunnett's test, $\mathrm{n}=3$ /group. EGFR, epidermal growth factor receptor.

A

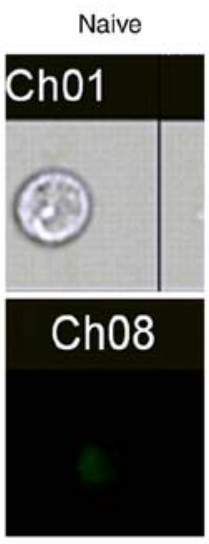

C

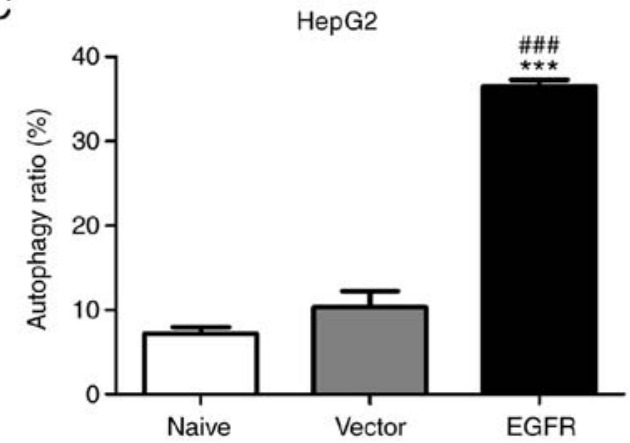

B
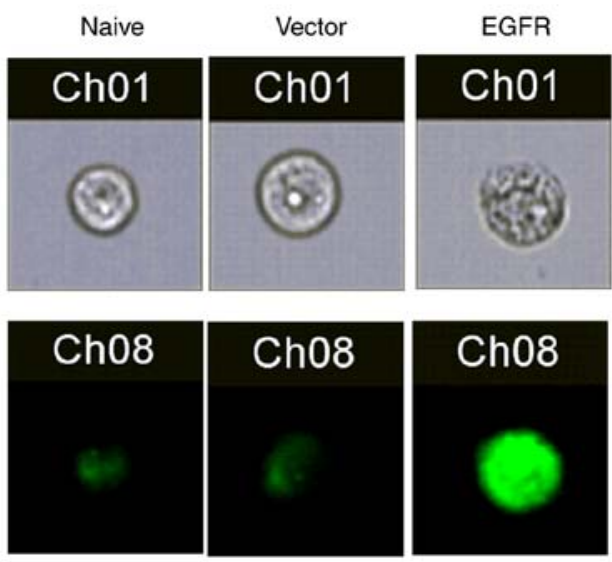

D

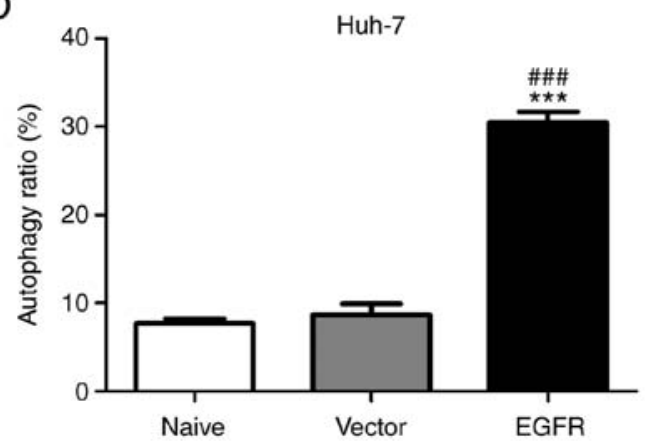

Figure 7. EGFR overexpression induces autophagy in HepG2 and Huh-7 cells. Representative MDC staining of (A) HepG2 and (B) Huh-7 cells. Analysis of the autophagy ratio in EGFR overexpressed (C) HepG2 and (D) Huh-7 cells. EGFR-overexpression significantly increased the autophagy ratio in HepG2 and Huh-7 cells. Naïve cells were untreated. ${ }^{* * *} \mathrm{P}<0.001$ vs. vector group, ${ }^{\# \# \#} \mathrm{P}<0.001$ vs. naive group, one-way analysis of variance followed by a Dunnett's test, $\mathrm{n}=5 /$ group. EGFR, epidermal growth factor receptor.

was $0.31 \pm 0.05 \mathrm{~g}$, compared with $0.67 \pm 0.07 \mathrm{~g}$ in the control group (Fig. 10A and B). Additionally, YM201636 promoted the expression of EGFR total protein in allograft tumors; however, the phosphorylation levels of EGFR at Tyr ${ }^{1068}$ were not notably altered (Fig. 10C and D). In addition, visceral organ indices were also examined to evaluate adverse effects of YM201636.
No significant differences in the visceral organ indexes (heart, liver, spleen, lung and kidney) were observed in the YM201636 group compared with the control group (Fig. 10E). These results suggested that YM201636 may inhibit tumor growth without notable systemic toxicity at the dose applied in the present study. 
A
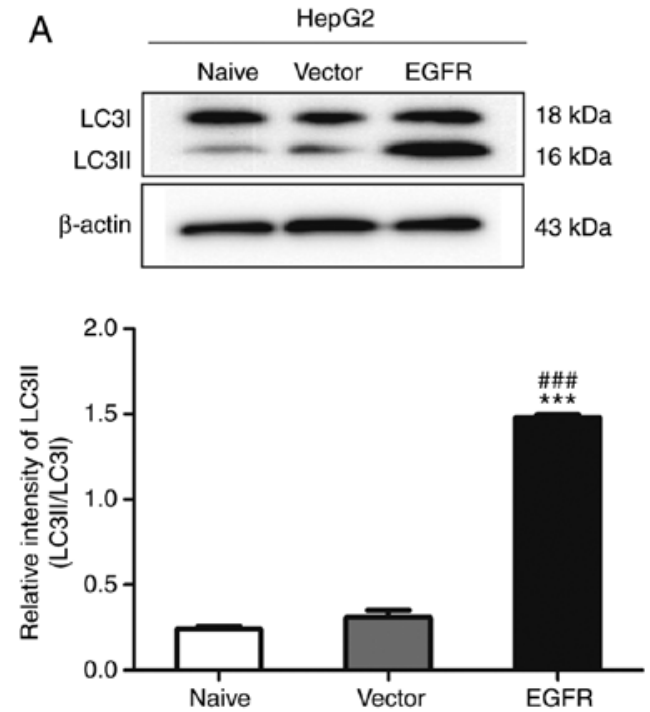

B
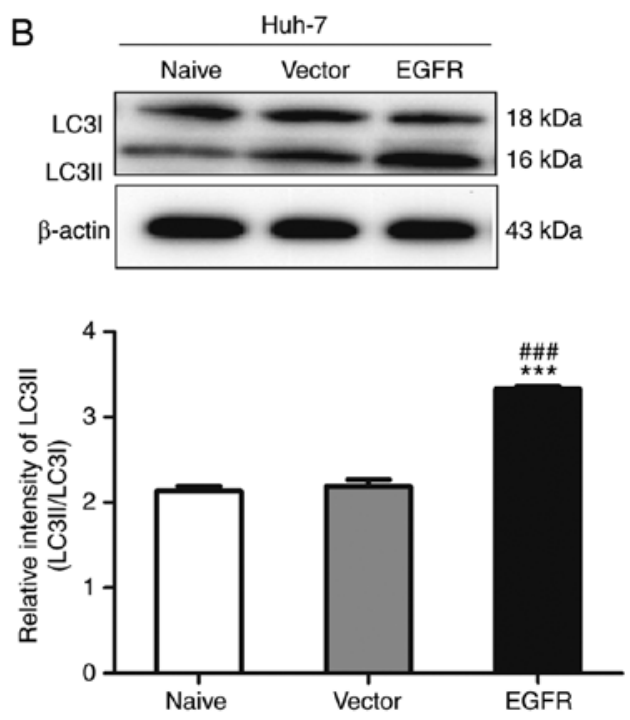

Figure 8. EGFR overexpression significantly increases the levels of LC3II. Western blot analysis of LC3 in (A) HepG2 and (B) Huh-7 cells. $\beta$-actin was used as an internal control. Naïve cells were untreated. ${ }^{* * *} \mathrm{P}<0.001$ vs. vector group, ${ }^{\# \# \#} \mathrm{P}<0.001$ vs. naive group, one-way analysis of variance, $\mathrm{n}=5 /$ group. EGFR, epidermal growth factor receptor; LC3, microtubule associated protein 1 light chain 3.
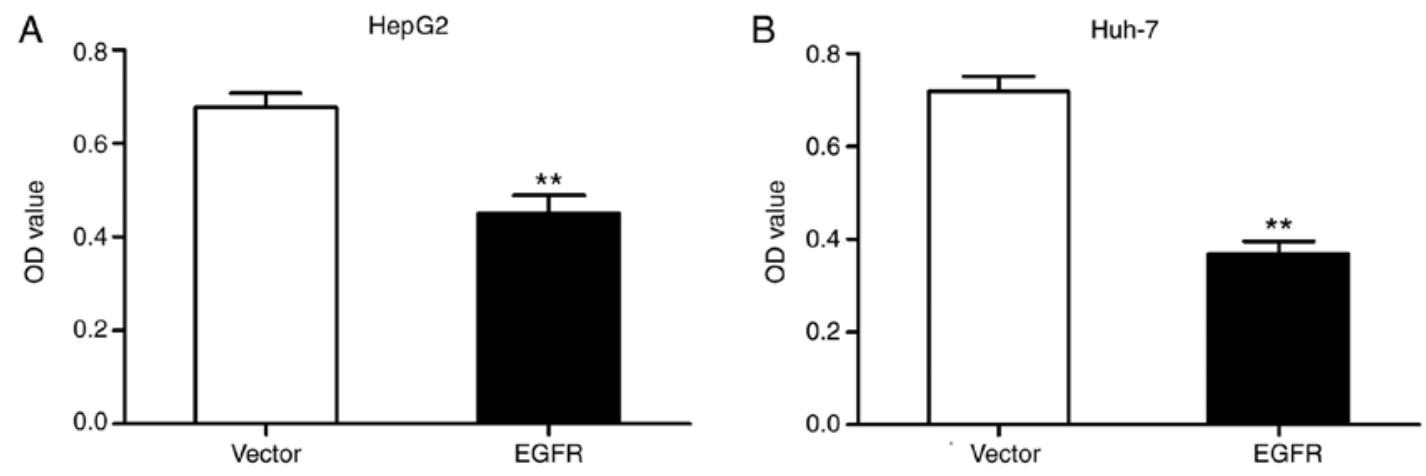

Figure 9. Overexpression of EGFR inhibits liver cancer cell proliferation. MTT assay in (A) HepG2 and (B) Huh-7 cells. ${ }^{* *} \mathrm{P}<0.01$ vs. vector group, two-tailed unpaired t-test, $\mathrm{n}=3$ /group. EGFR, epidermal growth factor receptor.

A

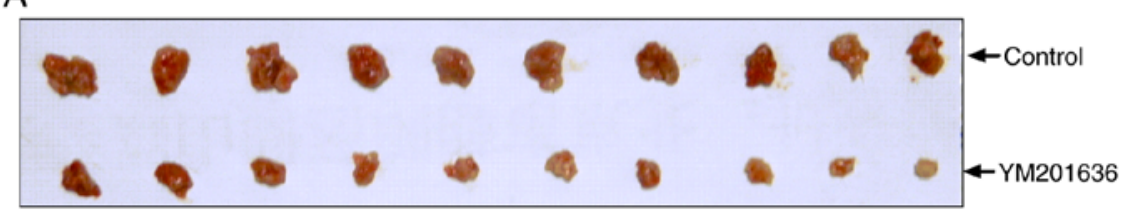

C

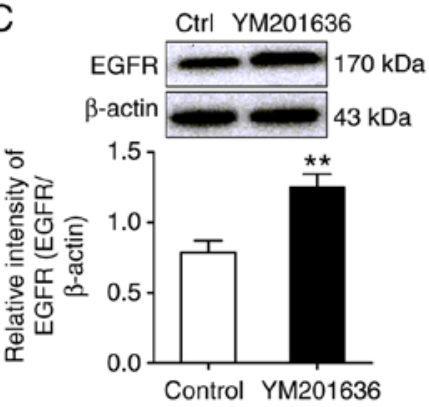

D

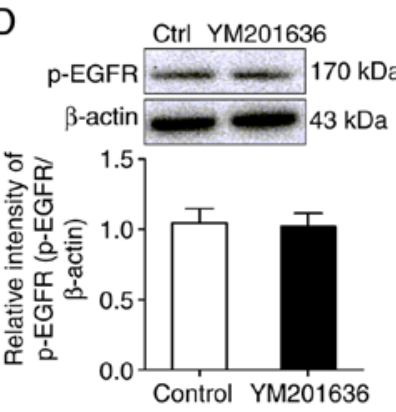

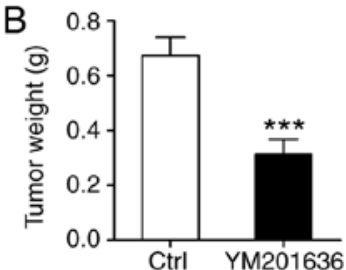

E

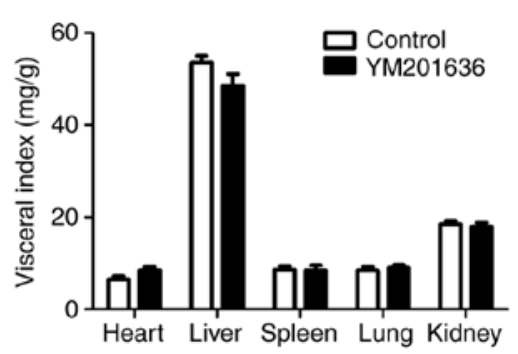

Figure 10. Antitumor activity of YM201636 in vivo. (A) Images of tumor obtained from the control and YM201636 groups. (B) Weight of the tumors excised from the control and YM201636 groups. YM201636 inhibits the tumor growth in tumor-bearing mice. ${ }^{* * *} \mathrm{P}<0.001$ vs. control group, two-tailed unpaired $\mathrm{t}$-test, $\mathrm{n}=10$ /group. (C) Western blot analysis of total protein expression of EGFR in xenograft tumors. ${ }^{* *} \mathrm{P}<0.01$ vs. control group, two-tailed unpaired t-test, $\mathrm{n}=5$ /group. (D) Phosphorylation of EGFR (Tyr ${ }^{1068}$ ) in YM201636 and control group tumors. $\mathrm{n}=5$ /group. (E) Visceral organ indices (heart, liver, spleen, lung and kidney) evaluated following treatment with YM201636. No significant difference was observed in the visceral organ indexes between the control and YM201636 groups (n=10). EGFR, epidermal growth factor receptor; p-, phospho-. 


\section{Discussion}

PIKfyve is an enzyme that is crucial for the synthesis of $\operatorname{PtdIns}(3,5) \mathrm{P} 2$ and has been associated with various membrane transport events $(20,21)$. Perturbations in the functions of PIKfyve lead to the formation of swollen endosomal/lysosomal structures in a variety of mammalian cell lines $(22,23)$. Additionally, PIKfyve has been proposed to be involved in oncogenesis and cancer cell migration. For example, PIKfyve promotes cell migration and invasion through the activation of Ras-related protein Raclin lung carcinoma, osteosarcoma and rhabdomyosarcoma cells $(8,24)$; knockdown of PIKfyve resulted in significant decreases in cell migration velocity and persistence (10). Consistent with these observations, in the present study, it was demonstrated that pharmacological inhibition of PIKfyve using YM201636 resulted in an inhibitory effect on tumor cell growth via the induction of autophagy in hepatoma cell lines.

The present study demonstrated that EGFR upregulation required for autophagy induced by the inhibition of PIKfyve. In support of the results, Er et al (18) reported that suppression of PIKfyve activity reduces the rate of EGFR degradation in MCF-10A cells. EGFR is part of the ErbB family of receptor tyrosine kinases and is overexpressed in many human cancers (25). Abnormal hyperactivation of EGFR is associated with unregulated proliferation, malignant transformation and metastasis in cancer cells $(26,27)$. It has been demonstrated that EGFR activation inhibits autophagy, dependent upon its kinase domain, by maintaining high activation levels of the $\mathrm{PI} 3 \mathrm{~K} /$ protein kinase $\mathrm{B} /$ mammalian target of rapamycin signaling pathway; knockdown or inhibition of EGFR signaling induces autophagy in various epithelial cancers $(28,29)$. However, in the present study, overexpression of EGFR induced autophagy in liver cancer cells. The results suggested that the increased EGFR may be inactive, as Tan et al (19) reported that inactive EGFR complexes collaborate with lysosomal-associated transmembrane protein $4 \mathrm{~B}$ and exocyst complex component 2 at endosomes to disassociate Rubicon from Beclin 1, which in turn initiates autophagy. Autophagy exhibits a dual role that can induce a cell survival or cell death response $(30,31)$. In the present study, EGFR-mediated autophagy was proposed to underlie cancer cell death.

Numerous studies have suggested EGFR overexpression promotes tumor cell proliferation $(26,32)$; however, the findings of the present study indicated that PIKfyve inhibitor YM201636-induced autophagy was dependent on EGFR overexpression. As mentioned above, the increased EGFR total protein was inactive EGFR, and inactive EGFR complexes could initiate autophagy (19). In support of the present results, Lanaya et al (33) demonstrated that mice lacking EGFR in hepatocytes develop more hepatocellular carcinoma (33). However, there are limitations in this study. Although the results suggested the increased EGFR total protein was inactive EGFR, the expression of proteins downstream of EGFR, such as Akt, p-Akt, ERK, p-ERK, was not analyzed. The phosphorylation of Akt and ERK are indicators of EGFR activation $(34,35)$, therefore, the expression of these downstream proteins will be examined in future studies.
In the present study, it was speculated that YM201636 induced total EGFR expression through reducing the rate of EGFR degradation in HepG2 and Huh-7 cells. Since EGF stimulation causes EGFR degradation by delivery to the lysosomes (18), therefore, it was assumed that the unchanged phosphorylation levels of EGFR at Tyr ${ }^{1068}$, was due to lack of EGF stimulation.

In conclusion, the results of the present study suggested that the PIKfyve inhibitor YM201636 may possess therapeutic potential for the treatment of liver cancer. In addition, EGFR overexpression induced by a PIKfyve inhibitor contributes to autophagy, thus leading to an inhibitory effect on cancer cell growth.

\section{Acknowledgements}

Not applicable.

\section{Funding}

This study was supported by the National Science Foundation of China (grant nos. 81573465, 81772832 and 81701110), the Natural Science Foundation of Henan (grant no. 162300410039); and the Program for Science and Technology of the Department of Education of Henan Province (grant no. 16A350013).

\section{Availability of data and materials}

All data generated or analysed during this study are available from the corresponding author on reasonable request.

\section{Authors' contributions}

DF and SQX participated in the design of the study, data analysis and manuscript writing. JZH, ZQX, JN conducted MTT assays and flow cytometry, participated in the design of the study and data analysis. WL, XW, CL and HS performed the assays and analysis. All authors read and approved the final manuscript.

\section{Ethics approval and consent to participate}

All experiments were approved by the Ethics Committee of University of Henan University (Kaifeng, China).

\section{Patient consent for publication}

Not applicable.

\section{Competing interests}

The authors declare that they have no competing interests.

\section{References}

1. Lozano R, Naghavi M, Foreman K, Lim S, Shibuya K, Aboyans V, Abraham J, Adair T, Aggarwal R, Ahn SY, et al: Global and regional mortality from 235 causes of death for 20 age groups in 1990 and 2010: A systematic analysis for the global burden of disease study 2010. Lancet 380: 2095-2128, 2012. 
2. Yang $Y$, Nagano $H$, Ota $H$, Morimoto $O$, Nakamura $M$, Wada $H$, Noda T, Damdinsuren B, Marubashi S, Miyamoto A, et al: Patterns and clinicopathologic features of extrahepatic recurrence of hepatocellular carcinoma after curative resection. Surgery 141: 196-202, 2007.

3. Sbrissa D, Ikonomov OC and Shisheva A: PIKfyve, a mammalian ortholog of yeast Fab1p lipid kinase, synthesizes 5-phosphoinositides. Effect of insulin. J Biol Chem 274: 21589-21597, 1999.

4. Shisheva A: PIKfyve: The road to PtdIns 5-P and PtdIns 3,5- $\mathrm{P}_{2}$ Cell Biol Int 25: 1201-1206, 2001.

5. Takasuga S and Sasaki T: Phosphatidylinositol-3,5-bisphosphate: Metabolism and physiological functions. J Biochem 154: 211-218, 2013.

6. McCartney AJ, Zolov SN, Kauffman EJ, Zhang Y, Strunk BS, Weisman LS and Sutton MA: Activity-dependent PI(3,5) $\mathrm{P}_{2}$ synthesis controls AMPA receptor trafficking during synaptic depression. Proc Natl Acad Sci USA 111: E4896-E4905, 2014.

7. Kim J, Jahng WJ, Di Vizio D, Lee JS, Jhaveri R, Rubin MA, Shisheva A and Freeman MR: The phosphoinositide kinase PIKfyve mediates epidermal growth factor receptor trafficking to the nucleus. Cancer Res 67: 9229-9237, 2007.

8. Oppelt A, Haugsten EM, Zech T, Danielsen HE, Sveen A Lobert VH, Skotheim RI and Wesche J: PIKfyve, MTMR3 and their product PtdIns5P regulate cancer cell migration and invasion through activation of Rac1. Biochem J 461: 383-390, 2014.

9. Ikonomov OC, Filios C, Sbrissa D, Chen X and Shisheva A The PIKfyve-ArPIKfyve-Sac3 triad in human breast cancer: Functional link between elevated Sac3 phosphatase and enhanced proliferation of triple negative cell lines. Biochem Biophys Res Commun 440: 342-347, 2013.

10. Oppelt A, Lobert VH, Haglund K, Mackey AM, Rameh LE, Liestol K, Schink KO, Pedersen NM, Wenzel EM, Haugsten EM, et al: Production of phosphatidylinositol 5-phosphate via PIKfyve and MTMR3 regulates cell migration. EMBO Rep 14: 57-64, 2013.

11. Dupuis-Coronas S, Lagarrigue F, Ramel D, Chicanne G, Saland E, Gaits-Iacovoni F, Payrastre B and Tronchere H: The nucleophosmin-anaplastic lymphoma kinase oncogene interacts, activates, and uses the kinase PIKfyve to increase invasiveness. J Biol Chem 286: 32105-32114, 2011.

12. Sano O, Kazetani K, Funata M, Fukuda Y, Matsui J and Iwata $\mathrm{H}$ Vacuolin-1 inhibits autophagy by impairing lysosomal maturation via PIKfyve inhibition. FEBS Lett 590: 1576-1585, 2016

13. Hessvik NP, Overbye A, Brech A, Torgersen ML, Jakobsen IS, Sandvig K and Llorente A: PIKfyve inhibition increases exosome release and induces secretory autophagy. Cell Mol Life Sci 73 4717-4737, 2016

14. Galluzzi L,BaehreckeEH,Ballabio A,Boya P,Bravo-SanPedroJM, Cecconi F, Choi AM, Chu CT, Codogno P, Colombo MI, et al: Molecular definitions of autophagy and related processes. EMBO J 36: 1811-1836, 2017.

15. Lee YK and Lee JA: Role of the mammalian ATG8/LC3 family in autophagy: Differential and compensatory roles in the spatiotemporal regulation of autophagy. BMB Rep 49: 424-430, 2016.

16. Bhat P, Kriel J, Shubha Priya B, Basappa, Shivananju NS and Loos B: Modulating autophagy in cancer therapy: Advancements and challenges for cancer cell death sensitization. Biochem Pharmacol 147: 170-182, 2018.

17. Heckmann BL, Yang $X$, Zhang $X$ and Liu J: The autophagic inhibitor3-methyladenine potently stimulates PKA-dependen lipolysis in adipocytes. Br J Pharmacol 168: 163-171, 2013.

18. Er EE, Mendoza MC, Mackey AM, Rameh LE and Blenis J: AKT facilitates EGFR trafficking and degradation by phosphorylating and activating PIKfyve. Sci Signal 6: ra45, 2013.
19. Tan X, Thapa N, Sun Y and Anderson RA: A kinase-independent role for EGF receptor in autophagy initiation. Cell 160: 145-160, 2015.

20. Hirano T, Munnik T and Sato MH: Inhibition of phosphatidylinositol 3,5-bisphosphate production has pleiotropic effects on various membrane trafficking routes in Arabidopsis. Plant cell Physiol 58: 120-129, 2017.

21. Ikonomov OC, Fligger J, Sbrissa D, Dondapati R, Mlak K, Deeb R and Shisheva A: Kinesin adapter JLP links PIKfyve to microtubule-based endosome-to-trans-Golgi network traffic of furin. J Biol Chem 284: 3750-3761, 2009.

22. Jin N, Jin Y and Weisman LS: Early protection to stress mediated by CDK-dependent $\mathrm{PI} 3,5 \mathrm{P}_{2}$ signaling from the vacuole/lysosome. J Cell Biol 216: 2075-2090, 2017.

23. Bissig C, Hurbain I, Raposo G and van Niel G: PIKfyve activity regulates reformation of terminal storage lysosomes from endolysosomes. Traffic 18: 747-757, 2017.

24. Dayam RM, Sun CX, Choy CH, Mancuso G, Glogauer M and Botelho RJ: The lipid kinase PIKfyve coordinates the neutrophil immune response through the activation of the Rac GTPase. J Immunol 199: 2096-2105, 2017.

25. Du Z and Lovly CM: Mechanisms of receptor tyrosine kinase activation in cancer. Mol Cancer 17: 58, 2018.

26. Ye QH, Zhu WW, Zhang JB, Qin Y, Lu M, Lin GL, Guo L, Zhang B, Lin ZH, Roessler S, et al: GOLM1 modulates EGFR/RTK cell-surface recycling to drive hepatocellular carcinoma metastasis. Cancer Cell 30: 444-458, 2016.

27. Sainsbury JR, Farndon JR, Needham GK, Malcolm AJ and Harris AL: Epidermal-growth-factor receptor status as predictor of early recurrence of and death from breast cancer. Lancet 1 : 1398-1402, 1987.

28. Li X and Fan Z: The epidermal growth factor receptor antibody cetuximab induces autophagy in cancer cells by downregulating HIF-1alpha and Bcl-2 and activating the beclin 1/hVps34 complex. Cancer Res 70: 5942-5952, 2010.

29. Li H, You L, Xie J, Pan H and Han W: The roles of subcellularly located EGFR in autophagy. Cell Signal 35: 223-230, 2017.

30. Chen Y, Henson ES, Xiao W, Huang D, McMillan-Ward EM, Israels SJ and Gibson SB: Tyrosine kinase receptor EGFR regulates the switch in cancer cells between cell survival and cell death induced by autophagy in hypoxia. Autophagy 12: 1029-1046, 2016.

31. Jacob JA, Salmani JMM, Jiang Z, Feng L, Song J, Jia X and Chen B: Autophagy: An overview and its roles in cancer and obesity. Clin Chim Acta 468: 85-89, 2017.

32. Sato H, Yamamoto H, Sakaguchi M, Shien K, Tomida S, Shien T, Ikeda $\mathrm{H}$, Hatono M, Torigoe H, Namba K, et al: Combined inhibition of MEK and PI3K pathways overcomes acquired resistance to EGFR-TKIs in non-small cell lung cancer. Cancer Sci 109: 3183-3196, 2018.

33. Lanaya H, Natarajan A, Komposch K, Li L, Amberg N, Chen L, Wculek SK, Hammer M, Zenz R, Peck-Radosavljevic M, et al: EGFR has a tumour-promoting role in liver macrophages during hepatocellular carcinoma formation. Nature Cell Biol 16: 972-977, 2014.

34. Zhang Y, Wang L, Zhang M, Jin M, Bai C and Wang X: Potential mechanism of interleukin-8 production from lung cancer cells: An involvement of EGF-EGFR-PI3K-Akt-Erk pathway. J Cell Physiol 227: 35-43, 2012.

35. Zhang L, Li Y, Lan L, Liu R, Wu Y, Qu Q and Wen K: Tamoxifen has a proliferative effect in endometrial carcinoma mediated via the GPER/EGFR/ERK/cyclin D1 pathway: A retrospective study and an in vitro study. Mol Cell Endocrinol 437: 51-61, 2016. 